\title{
Chapter 26 \\ How to Deal with \\ Relativistic Heavy Ion Collisions
}

\author{
Rolf Hagedorn
}

\begin{abstract}
A qualitative review is given of the theoretical problems and possibilities arising when one tries to understand what happens in relativistic heavy ion collisions. The striking similarity between these and $p p$ collisions suggests the use of techniques similar to those used 5-12 years ago in $p p$ collisions to disentangle collective motions from thermodynamics. A very heuristic and qualitative sketch of statistical bootstrap thermodynamics concludes an idealized picture in which a relativistic heavy ion collision appears as a superposition of moving 'fireballs' with equilibrium thermodynamics in the rest frames of these fireballs. The interesting problems arise where this theoretician's picture deviates from reality: non-equilibrium, more complicated motion (shock waves, turbulence, spin) and the collision history. Only if these problems have been solved or shown to be irrelevant can we safely identify signatures of unusual states of hadronic matter as, for example, a quark-gluon plasma or density isomers.
\end{abstract}

\subsection{Introduction}

During the last 2 years when I was working with Johann Rafelski on the Statistical Bootstrap Model [1] in order to adapt it to describe hot nuclear matter, I came more and more often across people concerned with relativistic heavy ion collisions, and also slowly became acquainted with the literature of this field-only to become more and more aware of how similar its problems are to those encountered in the

Invited lecture at Quark Matter 1: Workshop on Future Relativistic Heavy Ion Experiments at the Gesellschaft für Schwerionenforschung (GSI), Darmstadt, Germany, 7-10 October 1980; circulated in the GSI81-6 Orange Report, R. Bock and R. Stock, editors; and as preprint CERNTH-3014 dated 5 January, 1981 available at http://cds.cern.ch/record/134307.

R. Hagedorn: (deceased) CERN-TH, 1211 Geneve 23, Switzerland

J. Rafelski ( $\square)$

Department of Physics, The University of Arizona, Tucson, AZ 85721, USA

(C) The Author(s) 2016 
beginning of particle physics. Of the many different theoretical models invented and applied in the development of hadron physics, there is one - the 'thermodynamical model' [2], which tries to describe just those aspects of high energy particle collisions which are most strikingly similar to the main ones of relativistic heavy ion collisions, namely, the many-body aspects with an intimate mixture of coherent collective and incoherent stochastic movements.

I think one can still claim that the thermodynamical model was successful when applied with care and precaution. The well-known 'large transverse momenta' do not invalidate this model; they belong to phenomena outside its range of validity, as I shall explain later. That there was success at all-one dared to apply statistical thermodynamics to two-body collisons of elementary (sic) particles-was due to the many degrees of freedom in the final states and, without doubt, also to the colliding 'elementary' particles being much less elementary than one thought 30 years ago. The analogy with relativistic heavy ion collisions becomes obvious when the 'elementary' particles are considered as bags [3] filled with quarks and gluons. If I anticipate here that the present form of the statistical bootstrap model has good reasons to claim that, in collisions with a few $\mathrm{GeV}$ per nucleon, the individual bags will melt into a single bag, then the analogy between a $p p$ collision and a relativistic heavy ion collision is perfect; remaining differences in the theoretical treatment of these collisions are quantitative, but not principal.

It is therefore not surprising that several ideas of the thermodynamical model have been independently rediscovered by people concerned with relativistic heavy ion collisions. With all this in mind, I have the courage to dig deep into the past and uncover a few forgotten things which may still be useful for today's relativistic heavy ion collisions. The rather explicit list of references should compensate for the extremely qualitative style of this talk.

Notation and abbreviations: We use energy units $\mathrm{MeV}$ and $\mathrm{GeV}$ and set

$$
\hbar=c=k=1 \text {. }
$$

We use the abbreviations the relativistic heavy ion collision for relativistic heavy ion collisions and SBM for statistical bootstrap model.

\subsection{Collective Motions}

To my knowledge, Weisskopf [4] was the first to apply thermodynamics to the emission of particles from excited nuclei. The situation was favourable to such an approach: the excitation energy was low and the compound nucleus was long lived enough to reach an equilibrium state.

One would think that this could no longer be true in elementary collisions or the relativistic heavy ion collision. Nevertheless, when the first pion producing $p p$ collisions were analyzed, Koppe [5] realized that they could be interpreted as pion 
evaporation from some hot object of elementary dimensions. To honour Koppe for this pioneering work, this model was called the 'Fermi statistical model' [6].

The hot dense object became known as a 'fireball'. Very soon it was discovered that a single fireball could not explain the momentum distribution of emitted particles; it was impossible to find, for any given event, a Lorentz frame in which the momentum distribution was isotropic. Indeed, this should not have been expected, since even if a single fireball had formed it would, in general, have a very high spin. Moreover, phase space calculations show that the actual anisotropy-a forward/backward jet in the centre-of-momentum frame-cannot be accounted for by assuming a single, high spin fireball [7]. This is easily understood: the initial state has very definite phase relations between its individual partial waves; a single fireball, even if considered as a statistical sum over spins, cannot reproduce these phase relations.

It was then found, with the help of ingeniously chosen variables [8], that two fireballs moving with large opposite velocities in the $\mathrm{CM}$ frame were a much better approximation of reality. Adding a third fireball, at rest in CM, substantially improved the picture [9] (and references therein). Of course, the two oppositely moving fireballs need not have the same mass nor the same speed, and the third could also have some velocity in the CM. Therefore, one should rather introduce mass and velocity distributions, but then why have just three fireballs? Why not sometimes one, sometimes two, and sometimes three or even more? Thus, one should also introduce a distribution for the number of fireballs. It seems that, in this way, one obtains so much freedom that one can fit everything. This is not so if some simple model assumptions are made which are based on observation and which are very restrictive. This was done in the thermodynamical model [2], which I shall briefly describe. It was designed to predict inclusive momentum distributions and branching ratios of particles produced in high energy $p p$ collisions, but it was later easily adapted to $\pi \mathrm{p}, \mathrm{Kp}$ [10], and $p A$ (even heavy nuclei) collisions [11]. Some of the simplifying assumptions may be grossly wrong if extended to the relativistic heavy ion collision. I shall come back to this.

The simplifying postulates were $[2,12]$ :

Postulate 1. In high energy collisions of hadrons, collective motions have only components in the direction of the collision axis. It is possible to find a continuum of comoving Lorentz frames (local rest frames) such that a comoving observer will, in his neighbourhood, see only thermal motion. Turbulence is absent.

Postulate 2. All the kinetic energy of the incoming particles, which disappears by decelerating hadronic matter, is adiabatically and locally converted into excitation energy (heat).

Postulate 1 is illustrated in Figs. 26.1, and 26.2 The first figure images are taken from my CERN lectures in 1971 [12], while the second are from recent theoretical articles on the relativistic heavy ion collision $[13,14]$. Figure 26.1 is a picture of the distribution at the moment of impact, while Fig. 26.2 show a time development, on left in a model [13] and on right in a simplified hydrodynamic calculation [14], the 

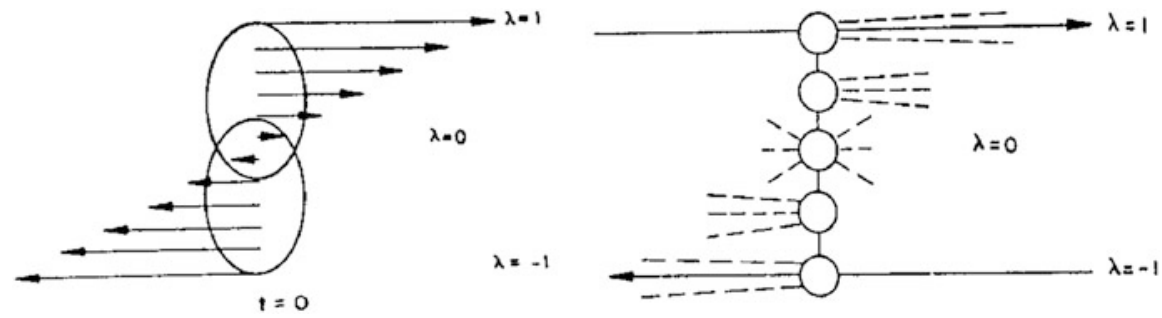

Fig. 26.1 Velocity distribution in a collision at the moment of impact, interpreted as continuous (on left) or as a probability distribution of fireballs (on right), from [12]
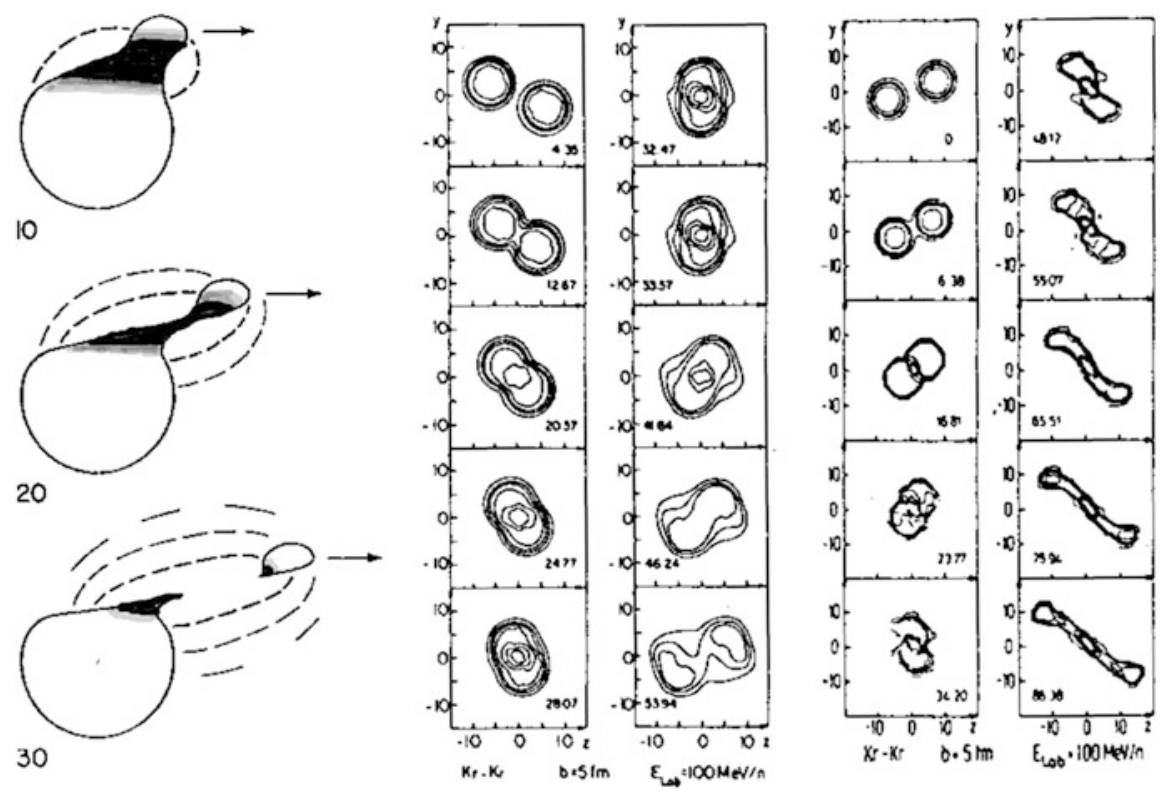

Fig. 26.2 On left: shape and velocity distribution in a collision of $250 \mathrm{MeV} /$ nucleon Neon on Uranium, seen at different times from top to bottom 10, 20, $30 \mathrm{fm} / \mathrm{s}$ after the moment of impact (qualitative figure follows Fig. 1 in [13]); on right: temporal development of density distribution in a low energy collision from a hydrodynamical calculation, the two sets of figures belong to different hydrodynamical assumptions, from [14]

two results are obtained under different assumptions about the equation of state from hydrodynamical calculations for a symmetric system. Some transverse collective motion is present in the latter case. 


\section{Useful Variables}

We use $\boldsymbol{\beta}=$ velocity and $\gamma=\left(1-\beta^{2}\right)^{-1 / 2}=$ Lorentz factor. A momentum fourvector is then $p=m(\gamma, \boldsymbol{\beta} \gamma)$. A Lorentz transformation along a given direction is fully determined by $\boldsymbol{\beta}$ or $\gamma$. It brings a particle from rest to velocity $\boldsymbol{\beta}$.

A very useful variable is the 'rapidity' $\eta$ defined by

$$
\gamma=\cosh \eta, \quad \beta \gamma=\sinh \eta, \quad \beta=\tanh \eta .
$$

Hence $\eta$ is the angle if a Lorentz transformation is represented as a rotation in Minkowski space. As the product of two rotations about the same axis is the rotation with the sum of the two angles, it follows for the product of two parallel and rotation free Lorentz transformations

$$
L\left(\eta_{2}\right) \circ L\left(\eta_{1}\right)=L\left(\eta_{1}+\eta_{2}\right)
$$

In applying Postulate 1, we shall have to ascribe at any moment to a fireball a velocity along the collision axis and, according to Postulate 2, an internal excitation equal to the kinetic energy which has disappeared by decelerating it from the initial to its present velocity. The excitation energy of a fireball must therefore be a function of the initial as well as of the actual velocity. Therefore, a suitable velocity variable should contain both the actual and the initial velocities. Giving the initial one a subscript zero, reasonable choices for velocity variables are then

$$
\lambda:=\frac{\sinh \eta}{\sinh \eta_{0}}, \quad \text { or } \lambda:=\frac{\eta}{\eta_{0}}, \quad \text { or } \lambda:=\frac{\gamma-1}{\gamma_{0}-1} \operatorname{sign}(\beta) .
$$

Note that $\lambda:=\beta / \beta_{0}$ is not suitable since $\beta$ and $\beta_{0}$ are almost always near to one and thus such a $\lambda$ would have no 'resolution power' for analyzing a relativistic velocity spectrum. The three other possible choices above all have good resolution power and they share the property $-1 \leq \lambda \leq 1$. The first choice makes $\lambda$ almost equal to Feynman's variable $x$ [15], while the second choice does not seem to have been used, and the third is still used today in the thermodynamical model $[2,16,17]$. It has the advantage of being physically obvious, since $(\gamma-1) /\left(\gamma_{0}-1\right)$ is the ratio of the actual kinetic energy density to the initial one of a decelerated volume element. However, it has the disadvantage of not being an analytic function as the other two are. Today I would prefer the first choice, but in this talk I leave the choice open. It could be any of the three or even some other one. 


\section{Momentum Distributions}

What is the situation now? We assume that there is a velocity distribution of hadronic matter which will depend on time $t$, space point $\mathbf{x}$, impact parameter $\mathbf{b}$, and 'velocity' $\lambda$ :

$$
\text { longitudinal velocity distribution } u(\lambda, \mathbf{x}, t, \mathbf{b}) \text {. }
$$

If properly normalized, $u(\lambda, \mathbf{x}, t, \mathbf{b}) \mathrm{d}^{3} x \mathrm{~d}^{3} b$ is the probability that the piece of matter contained in $\mathrm{d}^{3} x$ has, at time $t$, the velocity $\lambda$ when the impact parameter of the collision was in $\left\{\mathbf{b}, \mathrm{d}^{3} b\right\}$.

According to Postulate 1, such a piece of matter is, for a comoving observer, hot matter at rest in equilibrium, having a certain local temperature $T$. From very general arguments about black body radiation, it then follows that, in this volume element, the momentum distribution of particles with mass $m$ will be

$$
\mathrm{d}^{3} x f_{m}(\mathbf{p}, T) \mathrm{d}^{3} p=\frac{\mathrm{d}^{3} x}{(2 \pi)^{3}} \frac{\mathrm{d}^{3} p}{\exp \frac{\sqrt{p^{2}+m^{2}}}{T} \pm 1} .
$$

For $m=0$, this is just the Planck formula which initiated quantum physics.

The local temperature $T$ can now be calculated from Postulate 2 if an equation of state is known (see below). Consider a piece of incoming hadron matter. Before the collision, it has the rest energy density $\varepsilon_{0}$ of cold hadron matter. Now follow it as a comoving observer until it has decelerated from its initial Lorentz factor $\gamma_{0}$ to the actual Lorentz factor $\gamma$ at time $t$. For the comoving observer, it is still at rest, but now has rest energy density $\varepsilon$ because the initial kinetic energy has become excitation energy. Postulate 2 asserts that

$$
\varepsilon \gamma=\varepsilon_{0} \gamma_{0}
$$

Assuming the equation of state is known, we furthermore have

$$
\varepsilon=\varepsilon(T) .
$$

This can be inverted to give $T(\varepsilon)$, and since we have $\varepsilon=\varepsilon_{0} \gamma_{0} / \gamma$, it follows that $T=T\left(\lambda, \gamma_{0}\right)$.

Now put everything together to obtain the momentum distribution of particles of mass $m$ in any fixed Lorentz frame. To be definite, we may choose the CM frame of the collision:

$$
\left[W_{m}(\mathbf{p}) \mathrm{d}^{3} p\right]_{\mathrm{CM}}=\int_{0}^{2 R} \mathrm{~d}^{3} b \int \mathrm{d} t \mathrm{~d}^{3} x \int \mathrm{d} \lambda u(\lambda, \mathbf{x}, t, \mathbf{b}) L(\lambda)\left[f_{m}\left(T\left(\lambda, \gamma_{0}\right), \mathbf{p}^{\prime}\right) \mathrm{d}^{3} p^{\prime}\right] .
$$


This formula does the following: for fixed $\lambda, \mathbf{x}, t, \mathbf{b}$, the momentum distribution $f_{m}\left(T, \mathbf{p}^{\prime}\right) \mathrm{d}^{3} p^{\prime}$ in the local $\lambda$ rest frame is Lorentz transformed by $L(\lambda)$ to the $\mathrm{CM}$ frame and then the integrations sum up all these local contributions to yield $W_{m}(\mathbf{p})_{\mathrm{CM}}$.

We now observe that neither the local spectrum $f_{m}\left(T, \mathbf{p}^{\prime}\right)$ nor the Lorentz transformation depend on $\mathbf{x}, t, \mathbf{b}$. Therefore, we can immediately integrate over these variables and obtain a new weight function

$$
V F\left(\lambda, \gamma_{0}\right):=\int \mathrm{d}^{3} b \int \mathrm{d} t \mathrm{~d}^{3} x u(\lambda, \mathbf{x}, t, \mathbf{b}),
$$

where $V$ is the total interaction volume and Eq. (26.7) reduces to

$$
W_{m}(\mathbf{p}) \mathrm{d}^{3} p=\int_{-1}^{1} F\left(\lambda, \gamma_{0}\right) L(\lambda)\left[V f_{m}\left(T\left(\lambda, \gamma_{0}\right), \mathbf{p}^{\prime}\right) \mathrm{d}^{3} p^{\prime}\right],
$$

where $F\left(\lambda, \gamma_{0}\right)$ now picks up all contributions to a given $\lambda$ summed over the entire spacetime history and all impact parameters. This formula can be written in a manifestly covariant way.

In Eq. (26.9), everything is known except the weight function $F\left(\lambda, \gamma_{0}\right)$. As a probability distribution, it must obey

$$
\int_{-1}^{0} F\left(\lambda, \gamma_{0}\right) \mathrm{d} \lambda=\int_{0}^{1} F\left(\lambda, \gamma_{0}\right) \mathrm{d} \lambda=1
$$

We normalize it independently over each half interval in order to allow target and projectile to have different mass. Now $F\left(\lambda, \gamma_{0}\right)$ is normalized and defined over the $\gamma_{0}$ independent interval $\{-1,0 ; 0,1\}$. Hence, if $\gamma_{0}$ varies, $F$ can only change shape. One would like to choose the definition of $\lambda$ in such a way that $F\left(\lambda, \gamma_{0}\right)$ becomes a scaling function:

$$
\lim _{\gamma_{0} \rightarrow \infty} F\left(\lambda, \gamma_{0}\right)=F(\lambda)
$$

It has turned out that the choice made in the thermodynamical model [2], that is, $\lambda=(\gamma-1) /\left(\gamma_{0}-1\right)$, which was made with that aim, almost led to the desired behaviour of Eq. (26.11). From 10 to $1,000 \mathrm{GeV}$ (ISR), $F\left(\lambda, \gamma_{0}\right)$ did not detectably depend on $\gamma_{0}$ if fitted to experiments [18]. However, if any, then only one choice of $\lambda$ can lead to Eq. (26.11), because if $\lambda_{f}=f\left(\gamma, \gamma_{0}\right)$ does so, then any other choice $\lambda_{g}=g\left(\gamma, \gamma_{0}\right)$ will not, unless a function of $\lambda_{f}$ above. 


\section{Determination of the Weight Function $F\left(\lambda, \gamma_{0}\right)$}

One can have two attitudes:

1. Try to calculate $F\left(\lambda, \gamma_{0}\right)$ from some model ${ }^{1}$ :

- from Regge poles [19],

- from form factors [20],

- from relativistic kinematics [21, 22],

- from hydrodynamics [23],

- from Monte Carlo cascade calculations [24],

- from the Boltzmann collision equation [25],

- from any other models (I apologize to the authors not quoted due to my ignorance).

2. Find it by parametrization and fit to experiments. This was done by several authors [2, 17]. When fitted to pion production at one primary energy, the same $F(\lambda)$ gave good predictions for other different energies (up to ISR), for other secondaries ( $\pi, \mathrm{N}, \mathrm{Y}, \mathrm{K}, \overline{\mathrm{N}}, \overline{\mathrm{Y}}, \mathrm{d}, \overline{\mathrm{d}}, \mathrm{He}^{3}, \overline{\mathrm{He}}^{3}$, etc.) and for other projectiles and/or targets $[10,11]$. As $F(\lambda)$ was parametrized with only two parameters (remaining the same and constant for all these processes), one could say that $F(\lambda)$ was nearly (i.e., within the precision of the fit and the comparison of predictions with the data [11]) a universal function, although at ISR energies, the behaviour at $\lambda$ near zero suggested a violation of the desired form of scaling [18]. The various $F(\lambda)$ calculated from models differ among each other and from the empirical one, but never dramatically, except for a possible singularity at $\lambda=0$.

\section{Violations of the Postulates 1 and 2}

Our postulates worked rather well in particle physics, but they may fail in the relativistic heavy ion collision as follows:

\section{(a) Transverse Collective Motions}

The function $F(\lambda)$ is designed to represent only longitudinal collective motions. In principle, there should also be a function $G\left(\lambda_{\perp}\right)$ for transverse collective motions, or better still, a function $F(\lambda)$ with $\lambda$ representing three-dimensional collective motions. While in $p p$ collisions, this was not necessary; hydrodynamic calculations [23] for the relativistic heavy ion collision indicate the existence of non-negligible

\footnotetext{
${ }^{1}$ Not all the listed references set out to calculate $F\left(\lambda, \gamma_{0}\right)$, but the models yield information which can be interpreted in terms of such a function.
} 
(a)

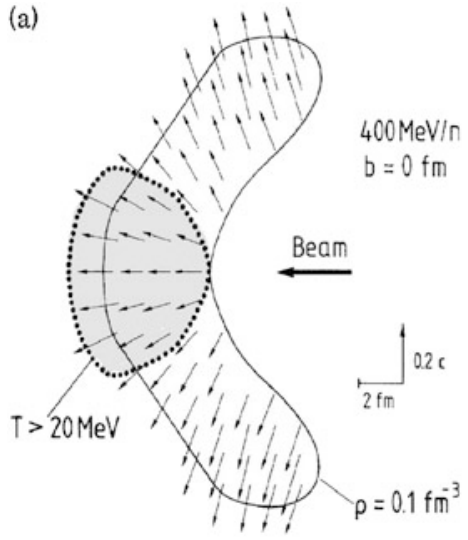

(b)

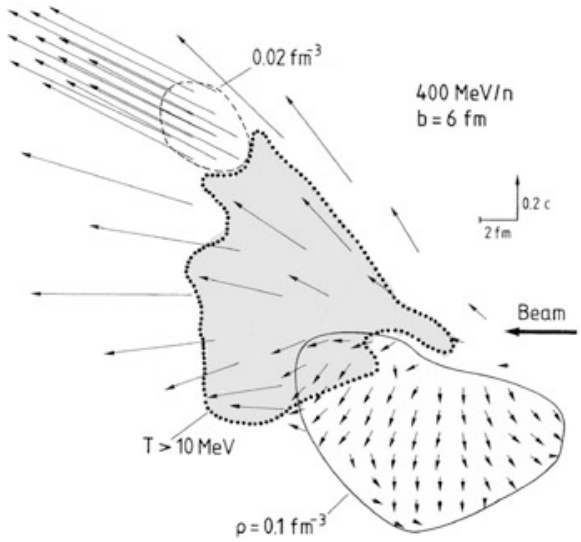

Fig. 26.3 Temperature, density, and velocity distributions in $400 \mathrm{MeV} /$ nucleon $\mathrm{Ne}-\mathrm{U}$ collisions, results from hydrodynamical calculations of [27]. Note the significant transverse velocity component

transverse components of hydrodynamic flow and of shock waves [26]. Figure 26.3 shows the result of a non-relativistic hydrodynamical calculation [27]. Clearly, all such calculations greatly depend on the assumptions made for the equations of state, viscosity, compressibility, and so on. As a child, I was much impressed when I discovered that solid cold tar was like a liquid if one had patience (a stone would sink into a tar barrel within a couple of days), but it would shatter like glass if hit hard. Thus transverse motion may depend on the collision energy.

Theoretical work in this domain can greatly profit from experiment; we will always have a superposition of collective and heat motion. Heat motion is (as we shall see) limited to typical values as, for example, at ISR energies [28]:

$$
\begin{aligned}
\left\langle P_{\perp}\right\rangle_{\text {proton }} & \approx 500 \mathrm{MeV} / c \Longrightarrow \beta_{\perp}^{(\mathrm{p})} \approx .47, \\
\left\langle P_{\perp}\right\rangle_{\pi} & \approx 350 \mathrm{MeV} / c \Longrightarrow \beta_{\perp}^{(\pi)} \approx .93 .
\end{aligned}
$$

Hence, the chance to observe transverse collective motion despite the thermal noise grows with the mass. For $m \gg T$, i.e., $m \gg m_{\pi}$, we have [2, 12]

$$
\left\langle p_{\perp}(m, T)\right\rangle \approx \sqrt{\frac{\pi m T}{2}} .
$$

Hence, with

$$
\begin{gathered}
\beta_{\perp}^{2}=\frac{\gamma_{\perp}^{2}-1}{\gamma_{\perp}^{2}}=\frac{p_{\perp}^{2}}{m^{2}+p_{\perp}^{2}}, \\
\left\langle\beta_{\perp}\right\rangle \approx \frac{\left\langle p_{\perp}\right\rangle}{m} \approx \sqrt{\frac{\pi T}{2 m}} \quad(m \gg T),
\end{gathered}
$$


the typical temperature for high collision energies is $T=160 \mathrm{MeV}$. This gives, with Eq. (26.15), for protons $\langle\beta\rangle \approx 0.52$, which shows that Eq. (26.15) already gives a good estimate for $m=m_{\mathrm{N}}$ and is rather good for heavier masses. Since even for very large collision energies $T \lesssim T_{0} \approx 160 \mathrm{MeV}$, we have for all energies above a few $\mathrm{GeV} /$ nucleon,

$$
\left\langle\beta_{\perp}\right\rangle_{A} \approx .52 / \sqrt{A}
$$

where $A$ is the nucleon number of the emitted fragment. For heavy fragments, the thermal transverse velocity $\beta_{\perp}$ is therefore small and may become smaller than the transverse collective velocity. Therefore, the transverse momentum of heavy fragments should be studied carefully because it allows one to determine the collective transverse motion and to compare it to hydrodynamic calculations. Turbulence might also be detected by such measurements.

\section{(b) Violation of Postulate 2}

Postulate 2 is also certainly violated and here it seems to be difficult to say how this could be experimentally detected because there are many violating mechanisms:

- It is not true that an incoming volume element will simply be decelerated. It will also be deformed and its matter content will be mixed by mutual interpenetration with that of the collision partner. Nevertheless, it seems that the velocity weight function $F\left(\lambda, \gamma_{0}\right)$ is able to absorb part of this type of violation and that the fast rise of the local temperature towards some limiting value does the rest to dissimulate it.

- While heating up matter, some particle emission already takes place. Thus, heating is not quite adiabatic. However, emission is damped by $\exp (-m / T)$ so that only near the highest temperature reached in the heating process will particle emission be significant.

- Equilibrium might not be established even locally. We will come back to this point.

- Heat conduction might take place [29]. This will be negligible if break-up and particle emission is faster than heat transfer.

- Reabsorption of emitted particles violates not so much Postulate 2 as the assumption made in Eq. (26.9) that emission in the local rest frame is isotropic. Figure 26.4a shows how this can generate an asymmetry in the angular distribution ('hot spot') [29]. Figure 26.4b shows that this asymmetry would also disappear if longitudinal break-up into many fireballs is fast. 

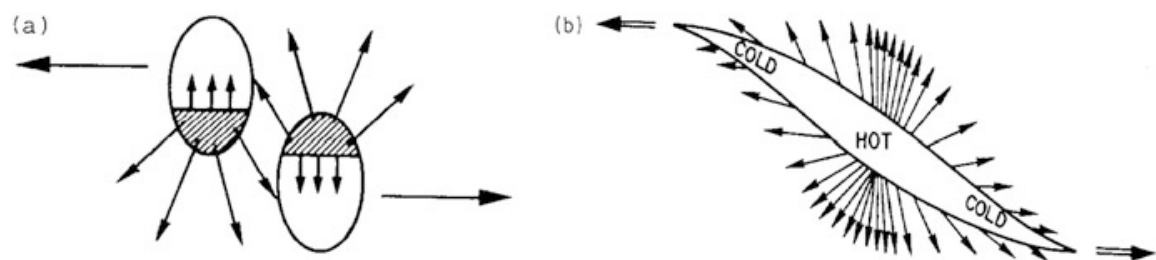

Fig. 26.4 Two extreme possibilities for the situation after a collision. (a) Two 'hot spots' leading to an asymmetry in the lab distribution of produced particles. (b) A fast stretching continuum will not lead to an asymmetry

\subsection{Statistical Bootstrap Thermodynamics}

The whole philosophy and all technical details of SBM are described in the literature $[1,12,30]$. Here I shall be very qualitative.

\section{The Partition Function}

Consider a microscopic system confined to a volume $V$ and embedded in a heat bath of temperature $T$. It will have an energy level spectrum $S=$ $\left\{E_{0}, E_{1}, E_{2}, \ldots, E_{n}, \ldots\right\}$. As an example, think of an ideal gas of one sort of particles of mass $m$. Then the probability of finding the system in the energy level $E_{n}$ is proportional to $\exp \left(-E_{n} / T\right)$. Normalised to one, we have

$$
W_{m}=\frac{\exp \left(-E_{n} / T\right)}{\sum_{i=1}^{\infty} \exp \left(-E_{i} / T\right)} .
$$

The expectation value of its energy is

$$
\begin{aligned}
\langle E\rangle & =\sum_{n=0}^{\infty} E_{n} W_{n}=\frac{\sum E_{n} \exp \left(-E_{n} / T\right)}{\sum \exp \left(-E_{n} / T\right)} \\
& =-\frac{\mathrm{d}}{\mathrm{d}(1 / T)} \ln \left[\sum_{n=1}^{\infty} \exp \left(-E_{n} / T\right)\right]
\end{aligned}
$$

The expression in square brackets is the partition function

$$
Z(T, V):=\sum_{n=1}^{\infty} \exp \left(-E_{n} / T\right)=: \int_{0}^{\infty} \sigma(E, V) \mathrm{e}^{-E / T} \mathrm{~d} E
$$


The density of states $\sigma(E, V)$ is defined by this identity; $\sigma(E, V) \mathrm{d} E$ is the number of energy levels in the interval $\{E, \mathrm{~d} E\}$. Equation (26.19) states that the partition function is the Laplace transform of the density of states.

From $Z(T, V, \ldots)$ all interesting thermodynamic quantities can be derived by logarithmic differentiation, as in Eq. (26.18). Apart from $T$ and $V$, the partition function may depend on further variables like chemical potentials (one for each conservation law), external fields, etc.

\section{Interaction}

We learn from chemists that, if there are atoms of sorts $A$ and $B$ which can undergo exothermic chemical reactions liberating the heat $Q$, viz.,

$$
A+B \rightleftarrows(A B)+Q
$$

where $(A B)$ is a molecule consisting of atoms $A$ and $B$, then one introduces just three different particles $A, B$, and $(A B)$ with masses $m_{A}, m_{B}$, and $m_{A B}$, with

$$
m_{A B}:=m_{A}+m_{B}-E_{\text {bind }}, \quad Q \equiv E_{\text {bind }} .
$$

If then no other sorts of particles and no other reactions occur, this is sufficient to calculate the chemical equilibrium rates $A: B:(A B)$ and the equations of state. One simply considers a three component ideal gas:

$$
Z(T, V, \ldots)=Z_{A}(T, V, \ldots) Z_{B}(T, V, \ldots) Z_{(A B)}(T, V, \ldots)
$$

and calculates everything from

$$
\ln Z=\ln Z_{A}+\ln Z_{B}+\ln Z_{(A B)} .
$$

We need not know any details except $E_{\text {bind }}$ about the interaction between $A$ and $B$, nor of the internal structures of $A, B$, and $(A B)$. The values of $m_{A}, m_{B}$, and $m_{A B}$ (which contains $E_{\text {bind }}$ ) are sufficient to represent the interaction for all questions about the equilibrium state (not, for example, for the question of how fast equilibrium is reached). This method can be pushed further. We could also include molecules $\left(A_{l} B_{k}\right)$ with $l, k=1,2, \ldots$ or add further elementary objects (atoms) $C, D, \ldots$ and consider molecules $\left(A_{i} B_{j} C_{k} D_{l} \ldots\right)$ as well as their excited states.

Back to particle physics. Here we include all possible reactions and all bound states, excited states, and resonances of the elementary input particles. The latter are chosen by convenience. One could start with quarks and gluons, but equally well with pions and nucleons (or with all these at the same time). Let us consider pions and nucleons (one could add strange, charmed, and other particles). 
Fig. 26.5 The mass spectrum $\rho(m)$ of hadrons (schematically). Each line represents one particle and their density grows exponentially with the mass

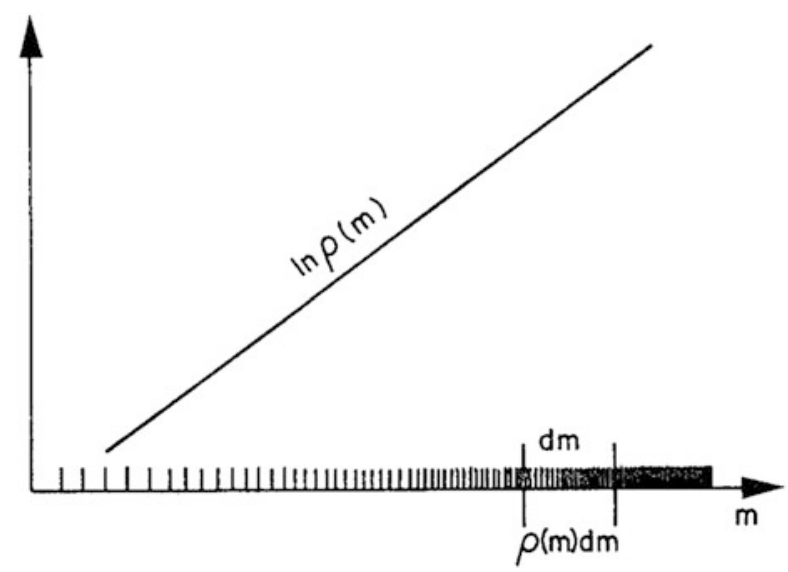

Figure 26.5 shows a mass distribution of $\pi$ and its resonances, $A$ and its resonances, and bound states (nuclei) of $(\pi \mathrm{N})$ and its resonances. We know from the chemists that we need all these masses and that we have only to consider a mixture of ideal gases, one for each particle mass (labelled $i=1, \ldots, \infty)$ :

$$
\ln Z(T, V, \ldots)=\sum_{i} \ln Z_{m_{i}}(T, V, \ldots)=: \int \mathrm{d} m \rho(m) \ln Z_{m}(T, V, \ldots)
$$

where $\rho(m) \mathrm{d} m$ is the number of different sorts of particles in $\{m, \mathrm{~d} m\}$, while $Z_{m}(T, V, \ldots)$ is the ideal gas partition function for an unrestricted number of particles of mass $m$, and dots indicate further variables (chemical potentials). The number of particles has to be unrestricted because:

- their total number is unrestricted due to particle creation and annihilation,

- the number of each sort changes via 'chemical' reactions, e.g.,

$$
\mathrm{N}+\mathrm{N} \longrightarrow \mathrm{N}+\mathrm{N}^{*}+2 \pi
$$

For the partition function of an ideal gas of an arbitrary number of particles, we find in any textbook

$$
\ln Z_{m}(T, V)=V f(m, T) \mathrm{e}^{-m / T}, \quad f(m, T) \underset{m \gg T}{\longrightarrow}\left(\frac{m T}{2 \pi}\right)^{3 / 2} .
$$

Note that the factor $\mathrm{e}^{-m / T}$ is missing in most textbooks, since in non-relativistic statistical mechanics it is an irrelevant normalizing factor. In the relativistic situation it is the important part as it governs the equilibrium between particle creation and annihilation. Hence,

$$
\ln Z(T, V)=V \int_{0}^{\infty} f(m, T) \rho(m) \mathrm{e}^{-m / T} \mathrm{~d} m
$$


is the partition function for the strongly interacting $\pi-\mathrm{N}$ gas, i.e., for the simplest strongly interacting hadron gas. What now is $\rho(m)$ ? We have the $\pi, \mathrm{N}$, all nuclei with their excited states, $\pi$ resonances, $A$ resonances, $\pi \mathrm{N}$ states and their resonances, etc. Only a finite number of them is known, but there are many more still unknown. The finite number of known states is, in general, sufficient to calculate some interesting quantities. This has been done for a long time-recently and in the context of nucleosynthesis in the early universe as well as for the relativistic heavy ion collision in some pioneering papers [31, 32]. In particular, the two papers by A.Z. Mekjan [32] are an excellent introduction to many fundamental concepts and open questions-most recommended reading!

What a finite number of states, included in the integral of Eq. (26.26) for $\ln Z$, cannot do, is to generate a singularity of the partition function, in other words, generate a phase transition. As one sees from Eq. (26.26), $\ln Z(T, V)$ is analytic in the entire right half of the complex $T$ plane if $O[\rho(m)]=m^{\alpha}, \alpha<\infty$. If $\rho(m)$ grows exponentially, $\rho(m) \sim C m^{\alpha} \exp \left(m / T_{0}\right)$, then the integral of Eq. (26.26) does not exist for $\operatorname{Re}(T)>T_{0}$ and $\ln Z(T, V)$ has a singularity at $T_{0}$, as first observed by Yu.B. Rumer [33], years before the SBM was proposed.

\section{The Bootstrap Hypothesis}

An incomplete $\rho(m)$, however, useful for computing low temperature properties of the system, will fail to exhibit critical phenomena. We therefore need the complete $\rho(m), 0 \leq m<\infty$. Indeed, only the complete spectrum can represent the full interaction; it is equivalent to the eigenphase representation of the S matrix [30].

We obtain the full mass spectrum from the 'bootstrap' hypothesis. The idea goes like this. From Eqs. (26.19) and (26.26), we have

$$
\begin{aligned}
& Z(T, V)=\int_{0}^{\infty} \sigma(E, V) \mathrm{e}^{-E / T} \mathrm{~d} E, \\
& Z(T, V)=\exp \left[V \int_{0}^{\infty} f(m, T) \rho(m) \mathrm{e}^{-m / T} \mathrm{~d} m\right] .
\end{aligned}
$$

The same function $Z(T, V)$ is expressed in different ways, once by the density of states of the whole system and once by the mass spectrum of its constituents.

We must clearly understand the physical meaning of $\sigma(E, V)$ and of $\rho(m)$ (see Fig. 26.6):

- $\sigma(E, V) \mathrm{d} E$ is the number of states between $E$ and $E+\mathrm{d} E$ of an interacting system enclosed in any externally given volume $V$.

- $\rho(m) \mathrm{d} m$ is the number of states (i.e., different particles) between $m$ and $m+\mathrm{d} m$ of an interacting system confined to its 'natural volume', i.e., to the volume resulting from the forces keeping these masses together as bound states or resonances. 
A macroscopic system

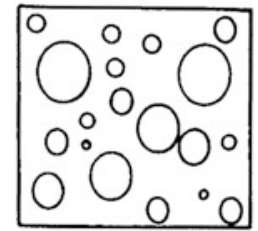

with total energy $E$ given volume $\mathrm{V}$ density of states $\sigma(E, V)$

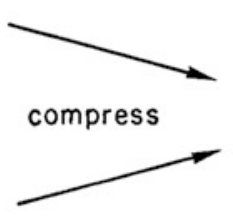

a "particle"

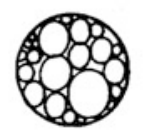

with total energy $\mathrm{m}$

self-confined to its

natural volume $V(m)$

density of states $\rho(m)$

Fig. 26.6 One step in the argument leading to the statistical bootstrap

Thus $\sigma(E, V)$ refers, in general, to some macroscopic system, while $\rho(m)$ refers to particles. Here the reader should hold on for a moment and imagine that we could compress the macroscopic system to that small volume which would be the natural volume $V(E)$ belonging to the energy $E$. What would happen? It would itself become a 'particle'-just one among the infinite number counted by the mass spectrum. Thus $\sigma(E, V(E))$ would have to be equal to the mass spectrum at $m=E$, namely, $\sigma(m, V(m))=\rho(m)$. This argument is so important that I will repeat it in another formulation:

The interaction reigning in the macroscopic system enclosed in $V$ is identical to the one creating the various bound states and resonances, keeping them together awhile and squeezing them into their natural volumes. On the other hand, we have claimed that the existence of all these-just exactly these! - bound states and resonances with all possible 'chemical' reactions between them, does represent—even generate—-this interaction. Hence,

the interaction is generated by reactions between bound states and resonances, which themselves are generated by the interaction, which is generated by reactions between bound states and resonances, and so on ad infinitum.

This circular reasoning is a special example of the more general bootstrap philosophy' proposed by G.F. Chew [34].

Coming back to the above gedankenexperiment, if we could compress the system with energy $E$ and volume $V$ to its natural volume $V(E)$, it would not be distinguishable from a resonance or a bound state with mass $m=E$ and volume $V(m)$. However, it then also follows that such a particle is also 'composed of' other particles just as it was before compressing and because it is subject to the same interaction (Fig. 26.6). Then of course $\sigma(E, V(E))$ is the number of states between 
$E$ and $E+\mathrm{d} E$ of a system confined to its natural volume; this is just the definition of $\rho(m)$ at $E=m$. Therefore, the function $\rho(m)$ is at the same time:

- the density of states of a composite system confined to its natural volume,

- and the mass spectrum of the constituents of such a system,

and $\rho$ generates the interaction which generates $\rho$. This double role of $\rho$ can be illustrated by a highly simplified 'bootstrap equation' in which everything except the double role has been omitted:

$$
\rho(m) \sim \rho\left(m_{1}\right) \rho\left(m_{2}\right) \ldots \rho\left(m_{N}\right), \quad \text { with } \sum_{i=1}^{N} m_{i}=m \text { for any } N
$$

Such a type of equation has only exponential solutions. Actually, the arguments are much more subtle and the equation for $\rho(m)$ is not as simple as Eq. (26.28), but the conclusion remains the same: $\rho$ is of the exponential type

$$
\rho(m)=g(m) \mathrm{e}^{m / T_{0}}, \quad O[g(m)]=m^{\alpha}, \quad \alpha<\infty,
$$

where $g(m)$ is not exponential. It is not easy to determine $g(m)$, but its asymptotic behaviour for $m \rightarrow \infty$ is well known. In fact, $g(m) \rightarrow C / m^{3}$. The reader will find more information in $[1,30,35]$. Here I mention only two things. The constants $C$ and $T_{0}$ can be guessed from a simplified model involving only pions [36]: $T_{0} \approx$ $m_{\pi}$. Such a spectrum fits well the lower part of the known spectrum of hadrons where we are sure to have found all resonances [1] and $T_{0}$ gives about the right slope. Furthermore, the same $T_{0}$ accounts quantitatively for the well-known limited mean transverse momenta of particles produced in high energy collisions because the partition function will become singular at $T_{0}$ (as explained above) and $T_{0} \approx m_{\pi}$ should be in some sense a limiting temperature or the critical temperature of a phase transition (boiling point of hadron matter). We shall come back to this.

Thus the bootstrap hypothesis allows one to predict the (averaged) hadronic mass spectrum and relates it to one of the most prominent features of high energy particle production-limited mean transverse momenta. It might be expected that it can also be applied to the relativistic heavy ion collision.

\section{The Singularity of the Partition Function: Baryon Conservation}

We have to conserve the baryon number in the relativistic heavy ion collision. So far we have ignored this, but now it will be built in. In order to do so, we must study the singularity of the partition function. We insert the exponential mass spectrum of Eq. (26.29) into Eq. (26.26) for $\ln Z$, combine the two non-exponential functions 
$f(m, T)$ of Eq. (26.26) and $g(m)$ of Eq. (26.29) into a new non-exponential function $h(m, T)$, and obtain

$$
\ln Z(T, V)=V \int_{0}^{\infty} h(m, T) \mathrm{e}^{m / T_{0}} \mathrm{e}^{-m / T} \mathrm{~d} m,
$$

where it is obvious that this integral exists for $T<T_{0}$ and has a singularity at $T=T_{0}$, the nature of which depends on $h(m, T)$ and does not interest us at the moment.

Now we split $\ln Z$ into two parts, $\ln Z_{\pi}$ and $\ln Z_{N}$, where the first one only contains pions and pionic resonances, while the second contains all baryonic states:

$$
\ln Z=\ln Z_{\pi}+\ln Z_{\mathrm{N}}
$$

where

$$
\ln Z_{\pi, \mathrm{N}}=V \int_{0}^{\infty} h_{\pi, \mathrm{N}}(m, T) \mathrm{e}^{m / T_{0}} \mathrm{e}^{-m / T} \mathrm{~d} m .
$$

Here we claim that the asymptotic part $\mathrm{e}^{m / T_{0}}$ of the mass spectrum is the same for pions and baryons. Qualitatively, this can be understood by considering all hadrons with a given baryon number $b$ and a very large mass such that $m \gg b m_{\mathrm{N}}$. For such large masses, the presence of a few baryons is irrelevant as most of the mass is due to excitation of non-baryonic degrees of freedom. Hence, for any fixed baryon number $b$, the asymptotic part of the mass spectrum must be the same and equal to the pionic one. This conclusion can be proved rigorously [37].

Consider now the baryonic partition function

$$
\ln Z_{\mathrm{N}}=V \int_{0}^{\infty} h_{\mathrm{N}}(m, T) \mathrm{e}^{m / T_{0}} \mathrm{e}^{-m / T} \mathrm{~d} m .
$$

The factor $\mathrm{e}^{-m / T}$ is proportional to the probability of creating a mass $m$ at temperature $T$. This factor is extremely small for small $T$ and so would be the number of baryons. If we wish $\ln Z_{\mathrm{N}}$ to exhibit a given number of brought-in baryons, we must counteract the small factor $\mathrm{e}^{-m / T}$. This can be done by artificially lowering $m$ by subtracting some suitable $\Delta m$ from it. This $\Delta m$ should account for the actual average baryon number $\langle b\rangle$ we wish to impose and it should lie between 0 and $m$ ( $\Delta m=0$ is no correction, $\Delta m=m$ is just excluded as too much). Thus we put

$$
\Delta m=m \frac{\mu}{\mu_{\mathrm{N}}},
$$


where $\mu$ is some parameter to be adjusted to yield the wanted $\langle b\rangle$. Then replacing $\mathrm{e}^{-m / T}$ by $\mathrm{e}^{-(m-\Delta m) / T}$, we obtain a new baryonic partition function

$$
\ln Z_{\mathrm{N}}(T, V, \mu)=V \int_{0}^{\infty} h_{\mathrm{N}}(m, T) \exp \frac{m}{T_{0}} \exp \left[-\frac{m}{T}\left(1-\frac{\mu}{m_{\mathrm{N}}}\right)\right] \mathrm{d} m .
$$

This partition function, in which $\mu$ can be chosen to give any desired expectation value $\langle b\rangle$ of the baryon number, has no longer an isolated singularity at $T_{0}$, but a singular curve in the $(T, \mu)$ plane. Indeed, it will be singular where the total exponent vanishes:

$$
T_{\text {crit }}=T_{0}(\mu):=T_{0}\left(1-\mu / m_{\mathrm{N}}\right) .
$$

This is the broken straight line in Fig. 26.7.

The above arguments are oversimplified in order to make the idea clear. In reality, one proceeds differently and $\ln Z_{\mathrm{N}}$ has a slightly different form, the critical curve in the $(T, \mu)$ plane is not trivial to calculate [35] and it looks like the curve in Fig. 26.7 instead of being a straight line. The partition function exists below that curve.

The new parameter $\mu$ introduced here (in a sloppy way) is called the chemical potential related to baryon number conservation. There is an extra chemical potential for every conserved quantity. Ours is the relativistic chemical potential. In non-relativistic statistical mechanics, it is defined as $\mu_{\mathrm{NR}}=\mu-m_{\mathrm{N}}$. This is consistent with omitting the factor $\mathrm{e}^{-m / T}$ in non-relativistic situations. Suppose we deal with a nucleon gas non-relativistically, then for the one-particle case

$$
E-\mu=\frac{p^{2}}{2 m_{\mathrm{N}}}+m_{\mathrm{N}}-\left(\mu_{\mathrm{NR}}+m_{\mathrm{N}}\right)=\frac{p^{2}}{2 m_{\mathrm{N}}}-\mu_{\mathrm{NR}}
$$

Fig. 26.7 The critical curve in the $(T, \mu)$ plane. The broken line is obtained from the handwaving 'derivation' in the text, while the full curve results from the model of [35]

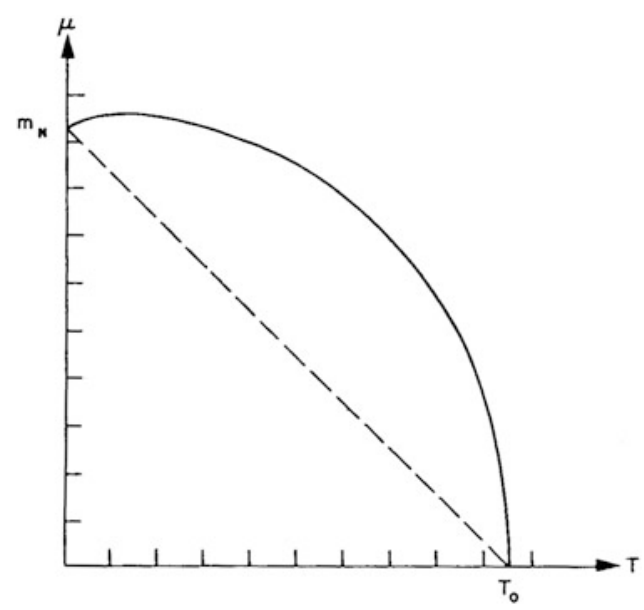


and

$$
\mathrm{e}^{-(E-\mu) / T}=\exp \left(-\frac{p^{2}}{2 m_{\mathrm{N}} T}+\frac{\mu_{\mathrm{NR}}}{T}\right)
$$

Thus the factor $\mathrm{e}^{-m_{\mathrm{N}} / T}$ has disappeared.

With the knowledge of $\rho(m)$ and the introduction of the chemical potential for baryon number conservation, $Z(T, V, \mu)$ has become calculable and is ready for application to the problem of highly excited hadronic (nuclear) matter. I stress that here I have only presented the general ideas. The complete analytical solution is technically more involved, but known in every detail [35].

\section{The Partition Function for Real (Extended) Particles}

All we have done so far suffers from a most unrealistic tacit assumption, namely, that our particles are pointlike. For dilute gases, this is known to be a good approximation. We, however, consider dense matter. Indeed, when applying the bootstrap argument, the system considered has the density of a composite particle, i.e., roughly nuclear density. From nuclear physics, we know that describing a nucleus as a gas of pointlike nucleons is a bad approximation. We also know that the volume of a nucleus is proportional to the number of its nucleons, i.e., to its total mass. In a relativistic situation, it is not possible to distinguish between mass due to the rest masses of constituents and mass due to kinetic energy. Hence, relativistically, the natural volume $V(m)$ of a particle must be proportional to the mass $m$, viz.,

$$
V(m)=\frac{m}{4 \mathscr{B}},
$$

where $\mathscr{B}$ is a fundamental constant with the dimension of energy density. Relation Eq. (26.37) is borne out not only by low energy nuclear physics, but also by bag models $[3]^{2}$ and by the statistical bootstrap model, as can be seen as follows:

- First write Eq. (26.37), which is valid in the particle's rest frame, in covariant form

$$
V^{\mu}(m)=\frac{p^{\mu}}{4 \mathscr{B}},
$$

\footnotetext{
${ }^{2}$ We take $\mathscr{B}$ to be the 'bag constant'. Then $4 \mathscr{B}$ is the energy density of a bag.
} 
by which the four-volume $V^{\mu}$ is defined. In the rest frame, this reduces to (26.37) and therefore is the unique generalization of (26.37) and of the corresponding low energy nuclear property.

- Consider a particle as a densely packed assembly of any number of other particles with masses $m_{i}$ :

$$
V^{\mu}(m)=\sum_{i} V^{\mu}\left(m_{i}\right), \quad \text { for any set }\left\{m_{1}, m_{2}, \ldots\right\}
$$

- Bootstrap tells us that the $m_{i}$ have the same composite internal structure as the composite $m$. Hence, the $V^{\mu}\left(m_{i}\right)$ must obey Eq. (26.38) with the same $\mathscr{B}$.

- Therefore,

$$
V^{\mu}(m)=\frac{p^{\mu}}{4 \mathscr{B}}=\frac{1}{4 \mathscr{B}} \sum_{i=1}^{N} p_{i}^{\mu},
$$

which is an identity, since $p^{\mu}=\sum_{i} p_{i}^{\mu}$ for any partition. This proves Eq. (26.37) to be true in statistical bootstrap.

Coming back to the partition function of a macroscopic system, we now introduce the notion of the available volume [35]

$$
\Delta:=V-\sum_{i=1}^{N} V\left(m_{i}\right)
$$

where $V$ is the externally given volume enclosing the system in a heat bath [for such a system, Eq. (26.38) is of course not true; it only holds for each of its constituents] and $\Delta$ is what remains after taking the proper volumes of all constituents away, just as in the van der Waals gas:

$\Delta$ is the volume in which the particles move as if they were pointlike, while in reality they have finite proper volumes and move in $V$.

There are some differences with the van der Waals gas, however:

1. The proper volumes $V\left(m_{i}\right)$ are not equal.

2. The proper volumes will have to be written covariantly

$$
V^{\mu}\left(m_{i}\right)=\frac{p_{i}^{\mu}}{4 \mathscr{B}}, \quad p_{i}^{\mu} p_{i \mu}=m_{i}^{2} .
$$

3. The usual factor four multiplying $\sum V_{i}$ is missing. It arises only for particles that are rigid spheres of equal radius. Our particles are deformable and of different sizes, in which case the factor is one. 
4. The second van der Waals correction, which simulates attractive forces by subtracting a density dependent term from the pressure, is not necessary here since bootstrap takes care of attractive forces (and to all orders in the virial expansion).

The above statement ( $\mathrm{vdW}$ ) implies that one obtains the partition function of real extended particles enclosed in $V$ by calculating the partition function of pointlike particles enclosed in the volume $\Delta$ :

$$
Z_{\text {real }}(T, V, \mu) \equiv Z_{\mathrm{pt}}(T, \Delta, \mu)
$$

Consider a particular microstate of the system where the particles have momenta $p_{i}^{\mu}$ $(i=1, \ldots, N)$. In that case, Eq. (26.41) reads

$$
\Delta^{\mu}=V^{\mu}-\sum_{i=1}^{N} \frac{p_{i}^{\mu}}{4 \mathscr{B}}=V^{\mu}-\frac{p^{\mu}}{4 \mathscr{B}},
$$

with $p^{\mu}$ being the momentary total four-momentum of the system ( $p^{\mu}$ fluctuates due to the heat bath). How then can we insert $\Delta$ in the partition function? The difficulties seem great since $Z_{\mathrm{pt}}$ is equal to $e^{Z_{1}}$, the one-particle function [see Eq. (26.27)], and yet we shall introduce a quantity which depends on all momenta and even fluctuates. We solve the problem by a tour de force. We choose $\Delta$ to be our independent volume-like parameter. Then

$$
V^{\mu}=\Delta^{\mu}+\frac{p^{\mu}}{4 \mathscr{B}}
$$

for any state contributing to $\ln Z$. Thus now $V$ is no longer fixed. It has, however, an expectation value. In the rest frame of the heat bath

$$
\langle V(E, \Delta)\rangle=\Delta+\frac{\langle E\rangle}{4 \mathscr{B}} .
$$

\section{Properties of the Real Hadron Gas}

From Eqs. (26.42) and (26.45), we can calculate all the usual thermodynamic variables. As an example, we calculate $\langle E\rangle /\langle V\rangle$, the energy density. Equation (26.18) says that

$$
\langle E\rangle=T^{2} \frac{\partial}{\partial T} \ln Z_{\text {real }}=T^{2} \frac{\partial}{\partial T} \ln Z_{\mathrm{pt}}(T, \Delta, \mu),
$$


and since $\ln Z_{\mathrm{pt}}(T, \Delta, \mu)$ is proportional to $\Delta$ [see, e.g., Eq. (26.35)],

$$
\langle E\rangle=\Delta\left[\frac{1}{\Delta} T^{2} \frac{\partial}{\partial T} \ln Z_{\mathrm{pt}}(T, \Delta, \mu)\right] .
$$

The expression in square brackets is the ( $\Delta$ independent) energy density of a gas of pointlike particles, viz., $\varepsilon_{\mathrm{pt}}(T, \mu)$. Hence,

$$
\langle E\rangle=\Delta \times \varepsilon_{\mathrm{pt}}=\left[\langle V(E, \Delta)\rangle-\frac{\langle E\rangle}{4 \mathscr{B}}\right] \varepsilon_{\mathrm{pt}}(T, \mu) .
$$

Here $\varepsilon_{\mathrm{pt}}$ does not depend on $\Delta$. Furthermore, $\Delta$ can be chosen so that any given value $\langle V(E, \Delta)\rangle$ is assumed $(\geq\langle E\rangle / 4 \mathscr{B})$. Hence we can now consider $\langle V\rangle$ as a variable which can be prescribed and we can thus solve Eq. (26.38) without regard for the implicit dependence of $\langle V\rangle$ on $\langle E\rangle$ :

$$
\langle E\rangle=\langle V\rangle \frac{\varepsilon_{\mathrm{pt}}(T, \mu)}{1+\varepsilon_{\mathrm{pt}}(T, \mu) / 4 \mathscr{B}}
$$

and

$$
\frac{\langle E\rangle}{\langle V\rangle}=\varepsilon_{\text {real }}(T, \mu)=\frac{\varepsilon_{\mathrm{pt}}(T, \mu)}{1+\varepsilon_{\mathrm{pt}}(T, \mu) / 4 \mathscr{B}} .
$$

Furthermore, from Eq. (26.48), $\langle E\rangle=\Delta \times \varepsilon_{\text {pt }}$, we find with Eq. (26.50),

$$
\langle V(T, \Delta, \mu)\rangle=\Delta\left[1+\frac{\varepsilon_{\mathrm{pt}}(T, \mu)}{4 \mathscr{B}}\right], \quad \Delta=\langle V\rangle\left[1-\frac{\varepsilon_{\mathrm{real}}(T, \mu)}{4 \mathscr{B}}\right]
$$

It turns out that all 'real' intensive quantities like pressure, baryon number density, etc., are related to the 'point' intensive quantities as $\varepsilon_{\text {real }}$ is related to $\varepsilon_{\mathrm{pt}}$ :

$$
\begin{aligned}
& \varepsilon_{\text {real }}(T, \mu)=\frac{\Delta}{\langle V\rangle} \varepsilon_{\mathrm{pt}}(T, \mu), \\
& P_{\text {real }}(T, \mu)=\frac{\Delta}{\langle V\rangle} P_{\mathrm{pt}}(T, \mu) \quad \text { (pressure), } \\
& v_{\text {real }}(T, \mu):=\frac{\langle b\rangle}{\langle V\rangle}=\frac{\Delta}{\langle V\rangle} v_{\mathrm{pt}}(T, \mu) .
\end{aligned}
$$




\section{Behaviour Near the Critical Curve}

Inspection of the exact partition function [35] reveals that $\varepsilon_{\mathrm{pt}}(T, \mu) \rightarrow \infty$ when the system approaches the critical curve. While, for given $T, \mu$, one can choose $\Delta$ to yield any given $\langle V\rangle \geq\langle E\rangle / 4 \mathscr{B}$ [see Eq. (26.45), Eq. (26.51) says how], the ratio $\Delta /\langle V\rangle$ is a definite function of $T$ and $\mu$, tending to zero when the system approaches the critical curve:

$$
\lim _{\text {(crit) }} \varepsilon_{\text {real }}(T, \mu)=4 \mathscr{B}
$$

that is, on the critical curve, the whole system assumes the density $4 \mathscr{B}$ of its constituents [remember Eq. (26.37)] and therefore has become just one giant 'particle'. Closer inspection [35] yields for the pressure and the baryon number density

$$
\lim _{\text {(crit) }} P_{\text {real }}(T, \mu)=0, \quad \lim _{\text {(crit) }} v_{\text {real }}(T, \mu)=v_{\text {crit }}(T, \mu) \neq 0, \infty .
$$

As the critical curve is reached at finite energy density, nothing prevents it being reached in actual particle collisions and nothing prevents it even being passed over, provided the collision was energetic enough. Considering hadrons as quark-gluon bags, the hadronic gas becomes then on the critical curve a giant quark-gluon bag, and it should be described as an interacting quark-gluon gas on the other side. This, at least, is our (J. Rafelski and R. Hagedorn) present interpretation [35] (Fig. 26.8).

Fig. 26.8 Physical interpretation of different regions of the $(T, \mu)$ plane as proposed in [35]

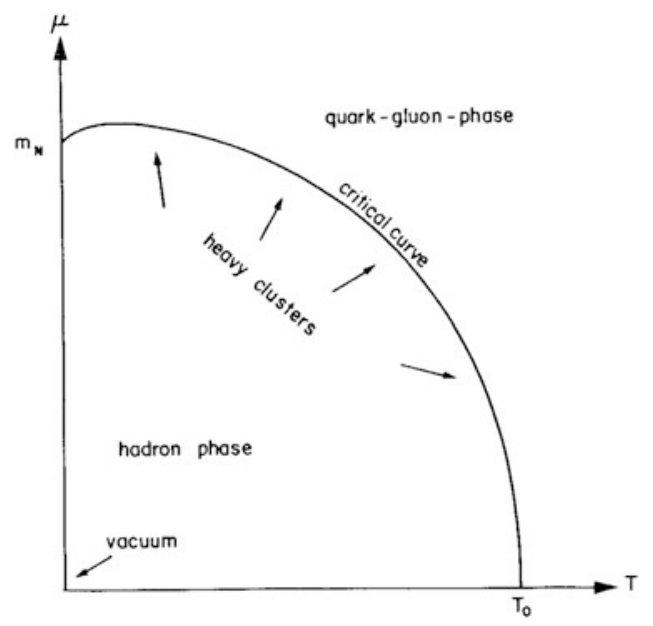


This thermodynamics, combined with a good description of the collective motions, should then give a model for the relativistic heavy ion collision:

$$
\text { Complete description }=\int\{\text { collective motion }\} \otimes\left\{\begin{array}{c}
\text { local bootstrap } \\
\text { thermodynamics }
\end{array}\right\} .
$$

While the local bootstrap thermodynamics is known, we still know little about the collective motions, which themselves depend again on the local thermodynamics. There remains a great deal of work to be done!

\subsection{Is There Equilibrium in the Relativistic Heavy Ion Collision?}

Nobody expects global equilibrium (except perhaps in selected 'central' collisions), but there are good reasons to doubt even that there is local equilibrium, because the duration of a collision, the lifetime of resonances, and the time needed to create a particle are all of the same order of magnitude. In particle collisions one can agree that thermodynamic equilibrium does not require a number of collisions of existing particles, but that the quantum mechanical probability distributions governing the creation of particles are such that the new-born particles seem to come from an equilibrium state [12]. This might be different in the relativistic heavy ion collision where many particles are already present before the collision and have to undergo collisions individually and/or coherently.

The argument for equilibrium seems to be valid in particle physics because the thermodynamical model which rests heavily on it was on the whole successful. With very few free parameters chosen once and for all, it covered collisions of different sorts of particles with lab energies between 10 and $1,000 \mathrm{GeV}$, describing rather well the features of particle production for all sorts of particles and with production rates ranging over 12 orders of magnitude. While many models are quantitatively superior in restricted areas, the thermodynamical model was (and still is) the only one covering the whole reasonably well. There are also some failures:

1. Two-particle correlations are not well described by the simple thermodynamical model [38].

2. The large $p_{\perp}$ processes observed at ISR energies [39] are not predicted by the model.

The second failure is particularly interesting because it concerns only about one per thousand of all produced particles - the rest behave as the model predicts. Figure 26.9 shows what happens qualitatively. The $p_{\perp}$ distribution takes off the straight line predicted by the model at $p_{\perp} \gtrsim 1.5 \mathrm{GeV} / c$ and stays higher up depending on the height of the collision energy. Why does this not kill the model? 


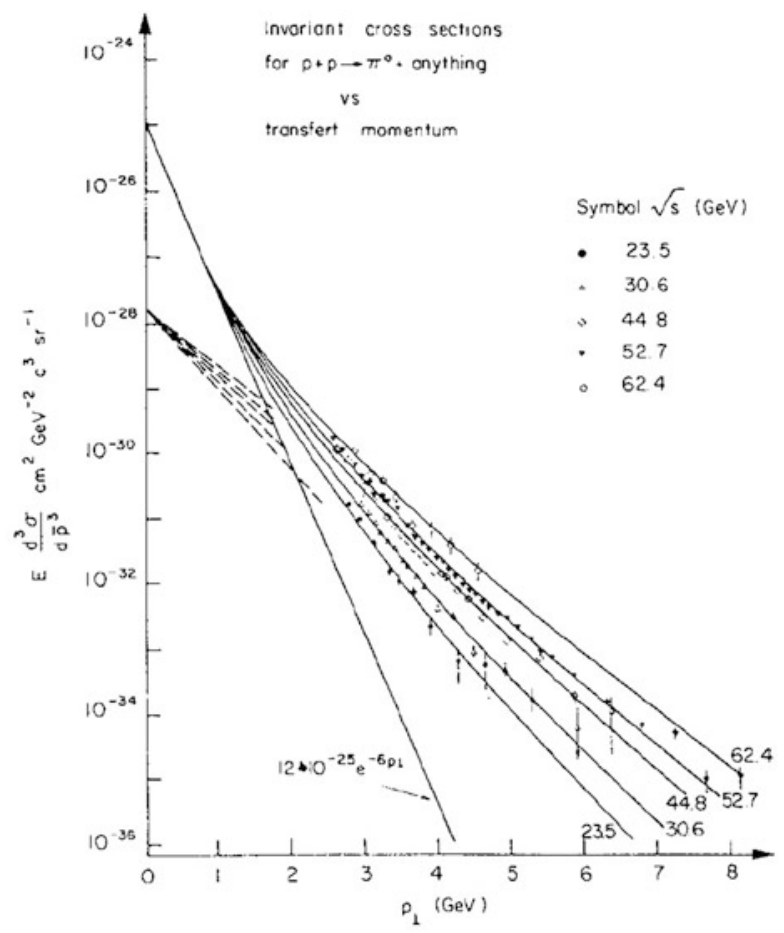

Fig. 26.9 Transverse momentum distribution of $\pi^{0}$ at ISR energies [39]. The curves (hand drawn by the present author) can be well approximated by a superposition of three exponentials. At $p_{\perp} \lesssim$ $1.5 \mathrm{GeV} c$, the SBM prediction holds with $T \approx 165 \mathrm{MeV}$, while at $p_{\perp} \gtrsim 5 \mathrm{GeV} / c$, a temperature growing $\approx E_{\mathrm{c} . \mathrm{m} \text {. }}^{2.7}$ (broken straight lines) suggests a plasma of gluons and not quite massless quarks

Because the straight parts of all curves, coinciding below $\sim 1.5 \mathrm{GeV} / c$ have the same energy-independent shape corresponding to a temperature $\sim 165 \mathrm{MeV}$, just as the model says. If the shape of this part had decreased steadily with rising energy, the model would have been in serious trouble. The large transverse momenta can be understood as being due to pre-hadronization processes taking place in the quarkgluon phase and emitting some energetic quark or gluon before crossing the critical curve. All the particles belonging to the straight line below $1.5 \mathrm{GeV} / c$ would then be emitted from the hadronic phase after crossing the critical curve.

While in $p p$ collisions at ISR energies there are thus definite traces of preequilibrium processes happening in the quark-gluon phase, such indications are as yet missing in proton-nucleus and nucleus-nucleus collisions, presumably because the collision energies are not yet high enough. In any case, models along the lines described in the previous sections have been applied to $p A$ and $A A$ collisions. I will mention just a few (apologizing to any authors not mentioned because of my ignorance): 
- The 'Black Book' on particle spectra [11] calculates the $p A$ collisions between 20 and $70 \mathrm{GeV}$ primary lab energy with $A$ being $\mathrm{Be}, \mathrm{Al}, \mathrm{Cu}$, and $\mathrm{Pb} . \mathrm{A}$ single collective velocity function $F(\lambda)$ covers all of these energies and targets in satisfactory agreement with data (where available). Production of heavy fragments is not calculated.

- J.P. Alard [40] pioneered the calculation of nucleon and heavy fragment emission $\left(\mathrm{He}^{4}, \mathrm{Be}, \mathrm{Li}, \ldots\right)$ using a special $F(\lambda)$ and introducing chemical potentials.

- A. Mekjian [41] calculated the relativistic heavy ion collision with $F(\lambda) \sim$ $\delta\left(\lambda-\lambda_{0}\right)$, where $\lambda_{0}$ was adjusted to represent a single moving fireball with thermodynamics restricted to the lower, explicitly known part of the spectrum.

- J. Gosset et al. [42] obtained good results using a velocity distribution ('fire streak') derived from kinematical considerations by W.D. Myers [13] and with thermodynamics using the lower part of the spectrum [31, 41].

- R. Malfliet [25] derived a collective velocity distribution [in the sense of $F(\lambda)$, but taking into account its temporal evolution] from the relativistic Boltzmann equation. Supplied with low-spectrum thermodynamics and nucleon-nucleon cross-sections, the model yielded particle spectra in good agreement with the relativistic heavy ion collision data.

All these attempts are based on the assumption of

$$
\{\text { local equilibrium }\} \otimes\{\text { collective motion }\}
$$

and they are all more or less successful. This is surprising.

\section{The Way to Equilibrium}

It is necessary to understand why there can be local equilibrium at least approximately. The problem has been considered in the literature [43-45]. Clearly, the approach to equilibrium takes time. After a sufficient time has elapsed, any system will come to equilibrium. What is less obvious is that the equilibrium state reached will, everything else being equal, depend on the volume available [12, 45]. Here I shall outline the ideas without going into detail. There are two kinds of equilibrium: kinetic and chemical:

- Kinetic equilibrium means equipartition of the total kinetic energy among the particles then present. This is a fast process which needs only very few collisions per particle. We assume this kind of equilibrium to be established instantaneously and locally, which means that a local temperature can be defined meaningfully. This temperature can still vary in space and time.

- Chemical equilibrium is equilibrium between the numbers of different species of particles. Being in equilibrium means for a given species that its rate of creation balances its death rate. To arrive at that state may take a short or a long time depending on cross-sections, lifetimes, densities, and so on. 
Consider a simplified example which exhibits the main point: a quasi-ideal pionnucleon gas in which a third kind of particle with a conserved charge $Q$ can be created. Let $\mathrm{A}$ and $\overline{\mathrm{A}}$ denote this particle and its antiparticle and $a$ the density of either $A$ or $\overline{\mathrm{A}}$, whence $a=N_{\mathrm{A}} / V$. Further, let $n$ be the number density of pions plus nucleons, viz., $n=\left(N_{\pi}+N_{\mathrm{N}}\right) / V$. Then

$$
\begin{aligned}
& \frac{\mathrm{d} a}{\mathrm{~d} t}=\left.\frac{\mathrm{d} a}{\mathrm{~d} t}\right|_{\text {creation }}-\left.\frac{\mathrm{d} a}{\mathrm{~d} t}\right|_{\text {annihilation }}, \\
& \left.\frac{\mathrm{d} a}{\mathrm{~d} t}\right|_{\text {creation }}=\sigma_{\mathrm{c}} v_{\mathrm{T}} n^{2} W_{\text {pair }} .
\end{aligned}
$$

Here $\sigma_{\mathrm{c}}$ is the inelastic cross-section (assumed energy independent and equal for all collisions $\pi \mathrm{N}, \pi \pi, A A), v_{\mathrm{T}}$ the mean thermal velocity, and $n$ the density of pions plus nucleons. We have assumed that $\mathrm{A}$ has a sufficiently large mass so that its contribution to $n$ is negligible: $n \gg a$ at all times. $W_{\text {pair }}$ is the pair creation probability per collision. Also,

$$
\left.\frac{\mathrm{d} a}{\mathrm{~d} t}\right|_{\text {annihilation }}=\sigma_{\mathrm{A}} v_{\mathrm{TA}} a^{2}
$$

where $\sigma_{\mathrm{A}}$ is the annihilation cross-section of $\mathrm{A}$ and $v_{\mathrm{TA}}$ is the mean thermal velocity of the A particles. Hence,

$$
\frac{\mathrm{d} a}{\mathrm{~d} t}=\sigma_{\mathrm{c}} v_{\mathrm{T}} n^{2} W_{\mathrm{pair}}-\sigma_{\mathrm{A}} v_{\mathrm{TA}} a^{2}=: \alpha-\beta a^{2}, \quad \alpha:=\sigma_{\mathrm{c}} v_{\mathrm{T}} n^{2} W_{\text {pair }}, \quad \beta:=\sigma_{\mathrm{A}} v_{\mathrm{TA}},
$$

where $\alpha, \beta$ are constants fixed by particle properties, densities, and temperature. Obviously equilibrium is reached when

$$
\frac{\mathrm{d} a}{\mathrm{~d} t}=0, \quad a(\infty)=\sqrt{\frac{\alpha}{\beta}} .
$$

The system approaches this value from below or from above depending on the initial value $a(0)$, as shown in Fig. 26.10. From the thermodynamical model, we know (first paper of [2] and CERN lectures [12]) that

$$
W_{\text {pair }} \sim e^{-2 m_{\mathrm{A}} / T},
$$

so that

$$
a(\infty)=\text { Const. } \times \mathrm{e}^{-m_{\mathrm{A}} / T}
$$


Fig. 26.10 The approach to equilibrium (qualitative) predicted by our simple model for two different initial values $a(0)$

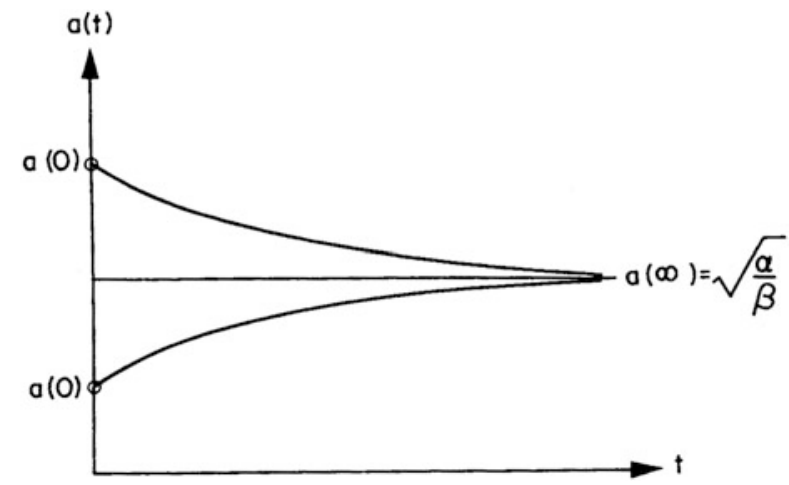

Consider now $a(0)$. This initial value is determined by the process which creates the $\pi \mathrm{N}$ gas in which the creation of $\mathrm{A} \overline{\mathrm{A}}$ pairs takes place. Let this process be an the relativistic heavy ion collision. Then in the first instant, $A A$ collisions at high (not thermal) velocities take place and, in these collisions, pions as well as $\mathrm{A} \overline{\mathrm{A}}$ pairs are created. Then kinetic equilibrium between all these is rapidly reached and Eqs. (26.56)-(26.58) can be applied, with $a(0)$ being the density of A resulting from the pairs created in the first impact, viz.,

$$
a(0) \sim \mathrm{e}^{-2 m_{\mathrm{A}} / T},
$$

the proportionality constant depending on the details of the collision (energy, number of nucleons, etc.).

Above, we have made the assumption that the density $a$ is so small that it can be neglected against $n$. This implies that after creation the particles $\mathrm{A}$ and $\overline{\mathrm{A}}$ part from each to large distances and that $\mathrm{A}(\overline{\mathrm{A}})$ has to wait for annihilation until colliding with some other particle $\overline{\mathrm{A}}(\mathrm{A})$. This, however, is only possible if the total volume available to the whole system is so large that the pairs created in the first instance can escape to distances which are large compared to the range of the annihilation interaction. If the volume were so small as to keep them always within annihilation distance, the number of pairs would always remain proportional to $\exp \left(-2 m_{\mathrm{A}} / T\right)$ as in $p p$ collisions $[2,12]$. Between such a small and a very large volume, many intermediate situations may occur in the relativistic heavy ion collision. Therefore, it is important to know the volume dependence of the equilibrium distribution $a(\infty)$, which may vary between $\sim \exp \left(-2 m_{\mathrm{A}} / T\right)$ and $\sim \exp \left(-m_{\mathrm{A}} / T\right)$ [45]. (Our above equation is valid only for large volumes, as $n \gg a$ was assumed.)

The time needed to reach equilibrium depends on the values of $\alpha$ and $\beta$, which determine the slopes of the curves in Fig. 26.10. Equilibrium is then reached when $|a(t)-a(\infty)|^{2}$ is of the order of the natural fluctuations in equilibrium, i.e.,

$$
|a(t)-a(\infty)|^{2} \underset{\text { equilbrium }}{\approx}\left\langle a^{2}\right\rangle-\langle a\rangle^{2}
$$


From this the equilibrium time can be determined [44].

\section{Expansion and Cooling}

Expansion and cooling after onset of a relativistic heavy ion collision are not equilibrium processes, but the only way presently known of describing them theoretically seems to be via a sequence of quasi-equilibrium states. Two approaches have been discussed in the literature [41, 43]: expansion with constant energy and with constant entropy. We believe [35] that constant entropy is wrong in early stage, while particle creation still takes place, no external work is done, and the process is irreversible. On the other hand, total energy is conserved, but it is insufficient to characterize the process, since we can calculate from thermodynamics only energy densities and do not know the dynamical expanding volume. On the other hand, baryon number is also conserved and must be taken into account. Again, we can only calculate the baryon number density and do not know the volume. The ratio of the two conserved quantities $\langle E\rangle$ and $\langle b\rangle$ is then also conserved and the unknown expanding volume drops out:

$$
\frac{\langle E\rangle}{\langle b\rangle}=\frac{\varepsilon}{v}=\left.\frac{E}{b}\right|_{\text {initial }}=\text { Const. }
$$

We therefore advocate the calculation of cooling curves given by (26.65) as a succession of quasi-equilibrium states [35]. We may visualize this as in Fig. 26.11 where we imagine the separating walls pulled down one after another, each time waiting until a new equilibrium is established. At some stage, pulling down more walls will not change any more the momentum distribution and particle ratios. We

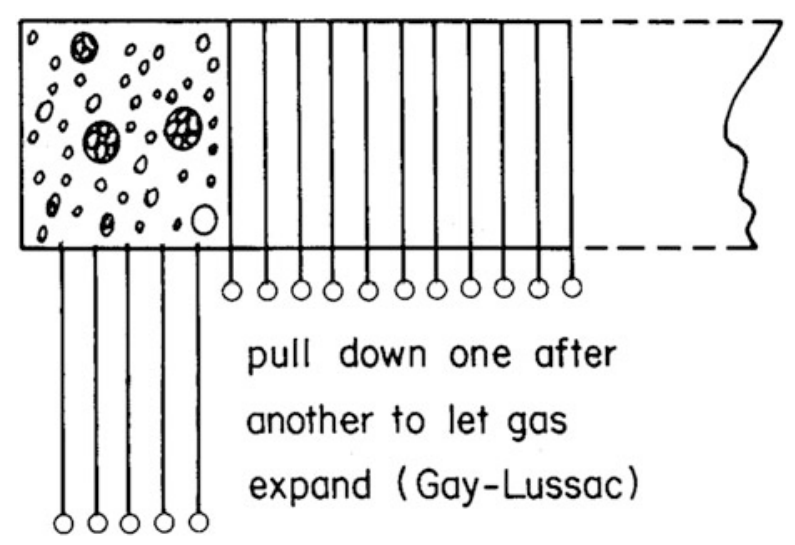

Fig. 26.11 Freely expanding hadron matter seen as a sequence of equilibrium states. Energy and baryon number are conserved, entropy increases 
have then reached an ideal gas situation: the equilibrium has been frozen [41, 43]. This state will be the one recorded on our particle detectors, but particles being emitted during the whole process will superimpose on it [35]. Clearly, the process pictured in Fig. 26.11 is only an approximation of reality, since equilibrium is not reached after each decay step. But it will be at first a much better approximation than that obtained from the assumption of constant entropy.

\subsection{Conclusions}

Despite a great number of well worked out partial theoretical models, we do not yet know enough to build a theory which describes coherently the whole of the relativistic heavy ion collision. Under these circumstances, even the detailed models cannot be adequately tested since we have not yet learned to disentangle the dozen or so different mechanisms mixed up in an the relativistic heavy ion collision. There exists, however, a fully worked out analogue computer programme based on the one and only true, complete theory: the relativistic heavy ion collision experiment. As we do not know the programme, but only its output for a given input, learning the theory from it is far from trivial. We should try to force it to give answers to the following unsettled questions:

- Is there a unique $F(\lambda)$ ?

- How important are transverse collective motions and turbulence?

- Must hydrodynamics be used?

- Is bootstrap thermodynamics right?

- How fast is equilibrium reached locally?

- Which is the best thermometer?

- How do fireballs cool and expand?

- Is there a phase transition to a quark-gluon plasma?

- Do ISR jets (large $p_{\perp}$ ) indicate such a phase transition?

These questions result from theoretical prejudice. Given that these prejudices might be reasonable to start with, the following experiments will be interesting:

1. Measure total multiplicities or relative ratios of secondaries: $\pi, \mathrm{N}, \mathrm{K}, \Lambda, \Sigma$, d, t, $\mathrm{He}^{3}, \mathrm{He}^{4}, \mathrm{Be}, \mathrm{Li}, \ldots$, and of as many of their antiparticles as feasible.

2. Measure for all of these the mean transverse momentum $\left\langle p_{\perp}\right\rangle$ as a function of the primary energy per nucleon $E / A$ and of the secondary's mass $m$.

For these first type types of measurement, try to make things as simple as possible: projectile $\equiv$ target and trigger for central collisions, in order to approach the theoretician's dream object—a single fireball.

3. Measure inclusive momentum distributions $W(\mathbf{p}) \mathrm{d}^{3} p$ as a function of $E / A$ and $m$. Try to fit with some $F(\lambda) \otimes$ \{local bootstrap thermodynamics $\}$ :

- Can one find an energy independent $F(\lambda)$ ? 
- Is it the same for all targets and projectiles?

- Is it independent of the emitted secondary?

- Does one need transverse collective motion?

For these measurements all events must be taken; triggering for central collisions or selecting events according to any specific criteria would distort the picture. One might start, however, by colliding equal nuclei and later make projectile $\neq$ target. Pay special attention to transverse momenta of heavy fragments, the heavier the better [see Eq. (26.16)].

4. Look for asymmetries in individual events:

- Azimuthal, i.e., non-isotropic in the angle about the collision axis. Such an asymmetry should arise from angular momentum conservation [46] and be large in peripheral, small in central collisions.

- While the first type of azimuthal asymmetry would still maintain symmetry with respect to reflection on the collision axis, even that might be destroyed by the 'hot spot' mechanism [29] leading to a right-left asymmetry.

5. Look for 'abnormal' things such as:

- sideward jets,

- unusually large $p_{\perp}$,

- unexpected (from equilibrium thermodynamics) particle ratios, in particular involving anti- $(\Lambda, \Sigma)$.

This last group of observations would have to be interpreted as evidence supporting the theoretical picture of a phase transition to a quark-gluon plasma. Any process originating in that phase and surviving the return to the hadron phase would leave traces of the sort mentioned.

Acknowledgements A great deal of what has been said here is due to a long and fruitful collaboration with J. Rafelski, without whom I might never have become interested in the relativistic heavy ion collision.

Open Access This book is distributed under the terms of the Creative Commons Attribution Noncommercial License which permits any noncommercial use, distribution, and reproduction in any medium, provided the original author(s) and sources are credited.

\section{References}

1. (A) Some of the more basic papers about SBM, its main ideas and difficulties:

R. Hagedorn, Suppl. Nuovo Cimento 3, 147 (1964);

R. Hagedorn, Nuovo Cimento 6, 311 (1968);

G. Matthiae, Nucl. Phys. B 7, 142 (1968);

R. Hagedorn, Nucl. Phys. B 24, 93 (1970);

K. Huang, S. Weinberg, Phys. Rev. Lett. 25, 855 (1970);

S. Frautschi, Phys. Rev. D 3, 2821 (1971); 
C.J. Hamer, S. Frautschi, Phys. Rev. D 4, 2126 (1971);

W. Nahm, Nucl. Phys. B 45, 525 (1972);

R.D. Carlitz, Phys. Rev. D 5, 3231 (1972);

I. Montvay, Phys. Lett. B 42, 466 (1972);

J. Yellin, Nucl. Phys. B 52, 583 (1973);

C. Hamer, Phys. Rev. D 8, 3558 (1973);

M.I. Gorenstein et al., Phys. Lett. B 43, 73 (1973);

H. Satz, Phys. Lett. B 44, 373 (1973);

E.M. Ilgenfritz, J. Kripfganz, Nucl. Phys. B 62, 141 (1973);

K. Fabricius, V. Wambach, Nucl. Phys. B 62, 212 (1973);

J. Engels, K. Fabricius, K. Schilling, Nuovo Cimento A 23, 581 (1974);

J. Kripfganz, E.M. Ilgenfritz, Phys. Lett. B 48, 329 (1974);

J. Engels, K. Fabricius, K. Schilling, Phys. Lett. B 53, 65 (1974);

F. Csikor, I. Farkas, Z. Katona, I. Montvay, Nucl. Phys. B 74, 343 (1974);

M.I. Gorenstein et al., Nucl. Phys. B 76, 453 (1974);

C.J. Hamer, Phys. Rev. D 10, 1458 (1974);

C.J. Hamer, Nucl. Phys. B 95, 173 (1975);

J. Engels, K. Fabricius, K. Schilling, Phys. Lett. B 59, 477 (1975);

S. Frautschi, Nucl. Phys. B 91, 125 (1975);

P. Fré, L. Sertorio, Nuovo Cimento A 28, 538 (1975);

J. Kripfganz, Nucl. Phys. B 100, 302 (1975);

N. Cabibbo, G. Parisi, Phys. Lett. B 59, 67 (1975);

D.E. Miller, Phys. Lett. B 64, 193 (1976);

E. Etim, R. Hagedorn, Nucl. Phys. B 131, 429 (1977);

M. Feigenbaum, L. Sertorio, Nuovo Cimento A 43, 31 (1978);

J. Letessier, A. Tounsi, H. Satz, Nuovo Cimento A 45, 590 (1978);

H. Satz, Phys. Rev. D 20, 582 (1979);

R. Fiore, Z. Phys. C 1, 305 (1979);

K. Redlich, L. Turko, Z. Phys. C 5, 201 (1980)

(B) For reviews and lectures see [12] and [30]

(C) See also $[25,35,36,45]$

2. R. Hagedorn, J. Ranft, Suppl. Nuovo Cimento 6, 169 (1968);

R. Hagedorn, Nuovo Cimento A 56, 1027 (1968); see also [17]

3. A. Chodos et al., Phys. Rev. D 9, 3471 (1974);

K. Johnson, Acta Phys. Pol. B 6, 865 (1975);

P. Hasenfratz, J. Kuti, Phys. Rep. C 40, 75 (1978)

4. V. Weisskopf, Phys. Rev. 52, 295 (1937);

R. Hagedorn, W. Macke, in Kosmische Strahlung, ed. by W. Heisenberg (Springer, Berlin, 1953)

5. H. Koppe, Z. Naturforsch. A 3, 251 (1948); Phys. Rev. 76, 688 (1949)

6. E. Fermi, Prog. Theor. Phys. 5, 570 (1950)

7. T. Ericson, Nuovo Cimento 21, 605 (1961);

F. Cerulus, Nuovo Cimento 22, 958 (1961);

H. Satz, Fortschr. Phys. 11, 445 (1963);

H. Joos, H. Satz, Nuovo Cimento 34, 619 (1964);

K. Zalewski, Acta Phys. Pol. 28, 933 (1965);

A. Kotanski, K. Zalewski, Nuovo Cimento 40, 134 (1965)

8. C. Castagnoli et al., Nuovo Cimento 10, 1539 (1953);

G. Cocconi, Phys. Rev. 111, 1699 (1958)

9. A. Jabs, Z. Phys. 222, 1 (1969)

10. H.R. Gerhold, B. Buschbeck, Nuovo Cimento A 22, 39 (1974);

K. Hänssgen, Application of the thermodynamical model to $\pi \mathrm{N}$ and $\mathrm{KN}$ collisions and charged and neutral strange particle production, Leipzig preprint KMU-HEP-7605 (1976) 
11. (A) Prediction and comparison of momentum spectra with experimental data for $p p$ and $p A$ collisions:

H. Grote, R. Hagedorn, J. Ranft, Atlas of Particle Spectra, CERN black report (1970)

(B) A variety of other predictions and/or confrontations with experiment:

S. Frautschi, C.J. Hamer, Phys. Rev. D 4, 2125 (1971);

F. Johns, B. Margolis, W.J. Meggs, R.K. Logan, Phys. Rev. Lett. 29, 756 (1972);

C.J. Hamer, Nuovo Cimento A 12, 162 (1972);

S. Frautschi, Nuovo Cimento A 12, 133 (1972);

B. Margolis, W.J. Meggs, R.K. Logan, Phys. Rev. D 8, 199 (1973);

G. Eilam, Y. Gell, B. Margolis, W.J. Meggs, Phys. Rev. D 8, 2871 (1973);

F. Csikor, I. Farkas, I. Montvay, Nucl. Phys. B 79, 92 (1974);

H.J. Möhring, J. Kripfganz, E.M. Ilgenfritz, J. Ranft, Nucl. Phys. B 85, 221 (1975);

H.J. Möhring, Acta Phys. Pol. B 9, 125 (1978);

H.J. Möhring, Nucl. Phys. B 87, 509 (1975);

C.B. Chiu, Phys. Rev. D 13, 2091 (1976);

S. Kogitz, R.K. Logan, S. Tanaka, Phys. Rev. D 14, 1420 (1976);

J. Vandermeulen, Bull. R. Sci. Liège 46, 57 (1977);

J. Vandermeulen, Contribution à l'étude phénoménologique de l'annihilation nucléon antinucléon, mémoire présenté Université de Liège, Faculté des Sciences (1976);

$\mathrm{S}$. Frautschi, Ericson fluctuations and the new Argonne data on $\pi \mathrm{N}$ scattering, in Hadron Matter at Extreme Energy Density, ed. by N. Cabibbo, L. Sertorio. Ettore Majorana International Science Series. Physical Sciences (1980);

R.K. Logan, M. Bates, S. Kogitz, Statistical bootstrap duality II, University Toronto preprint (1980);

see also $[11,12,17]$

12. R. Hagedorn, Cargèse Lecture in Physics, vol. 6 (Gordon \& Breach, New York 3, 1973), p. 643;

R. Hagedorn, Thermodynamics of strong interactions. CERN yellow report 71/12;

E.M. Ilgenfritz, J. Kripfganz, H.J. Möhring, Fortschr. Phys. 25, 123 (1977);

A. Tounsi, in Phenomenology at High Energies, Fourteenth Scottish Summer School, 1973 (London, 1974)

13. W.D. Myers, Nucl. Phys. A 296, 177 (1978)

14. H. Stöcker et al., GSI preprint 80-9 Darmstadt, Germany (1980)

15. R.P. Feynman, Phys. Rev. Lett. 23, 1415 (1969)

16. J. Ranft, 'SPUKJ' Programme W129 of the CERN programme library report CERN-LabIIRA-75-2; and Leipzig University report TUL 37-7 (1975)

17. J. Letessier, A. Tounsi, Nuovo Cimento A 11, 353 (1972); Nuovo Cimento A 13, 557 (1973); Nuovo Cimento A 15, 358 (1973);

J. Kripfganz, J. Ranft, Phys. Lett. B 55, 301 (1975);

J. Ranft, G. Ranft, Nucl. Phys. B 92, 207 (1975);

see also [2]

18. J. Hagedorn, J. Ranft, Nucl. Phys. B 48, 157 (1972); see also E. Celeghini, L. Lusanna, [22]

19. G. Ranft, J. Ranft, Phys. Lett. B 32, 207 (1970)

20. F. Cerulus, A liquid drop model for high energy elastic and inelastic scattering, in Proceedings of the Colloquium on High Multiplicity Hadronic Interactions (Ecole Polytech., Paris III Session, 1970)

21. J. Hüfner, J. Knoll, Nucl. Phys. A 290, 460 (1977)

22. E. Celeghini, L. Lusanna, Nucl. Phys. B 69, 58 (1974); see also [13, 20]

23. J. Hofmann, W. Scheid, W. Greiner, Nuovo Cimento A 33, 343 (1976); J. Hofmann, B. Müller, W. Greiner, Phys. Lett. B 82, 195 (1979)

24. J. Cugnon, T. Mizutani, J. Vandermeulen, University Liège preprint (1980);

J. Cugnon, T. Mizutani, J. Vandermeulen, Equilibration in rel. nucl. coll.; a Monte Carlo calculation. Caltech preprint MAP-10 (1980);

J. Cugnon, Non-equilibrium aspects in rel. nucl. coll. Caltech preprint MAP-15 (1980);

J. Cugnon, S.E. Koonin, Pion spectra and the geometry of nucl. coll. Caltech preprint MAP-17 (1980) 
25. R. Malfliet, Phys. Rev. Lett. 44, 864 (1980); R. Malfliet, Non-equilibrium dynamics in high energy nucleus-nucleus collisions I and II. Groningen preprint KVI-270 (1980)

26. H.G. Baumgardt et al., Z. Phys. A 273, 359 (1975); see also [23]

27. H. Stöcker, J.A. Maruhn, W. Greiner, Phys. Rev. Lett. 44, 725 (1980)

28. G. Giacomelli, M. Jacob, Phys. Rep. 55, 1 (1979)

29. R.M. Weiner, Phys. Rev. Lett. 32, 630 (1974);

R. Weiner, M. Weström, Nucl. Phys. A 286, 282 (1977);

R. Weiner, M. Weström, Phys. Rev. 34, 1523 (1975);

R.M. Weiner, Phys. Rev. D 13, 1363 (1976)

30. L. Sertorio, Riv. Nuovo Cimento 2, 1 (1979) (a review stressing fundamental aspects)

31. J.I. Kapusta, Phys. Rev. C 16, 1493 (1977)

32. A.Z. Mekjian, Phys. Rev. C 17, 1051 (1978); A.Z. Mekjian, Nucl. Phys. A 312, 491 (1978)

33. Yu.B. Rumer, J. Exp. Theor. Phys. 38, 1899 (1960)

34. G.F. Chew, S. Mandelstam, Phys. Rev. 119, 467 (1960);

F. Zachariasen, Phys. Rev. Lett. 7, $112(1961)$;

G.F. Chew, S. Frautschi, Phys. Rev. Lett. 7, 394 (1961);

G.F. Chew, M. Gell-Mann, A.H. Rosenfeld, Sci. Am. 210, 74 (1964);

G.F. Chew, Science 161, 762 (1968); ibid, Phys. Today 23, 23 (1970)

35. R. Hagedorn, J. Rafelski, Phys. Lett. B 97, 136 (1980); See the following Chapter 27 in this volume see also [1]

36. R. Hagedorn, I. Montvay, Nucl. Phys. B 59, 45 (1973);

E.M. Ilgenfritz, J. Kripfganz, Nucl. Phys. B 56, 241 (1973)

37. R. Hagedorn, J. Rafelski, Manuscript in preparation for Physics Reports (This manuscript was never completed, however, the material we developed is now presented in this volume drawn from different conference reports.)

38. G. Ranft, J. Ranft, Nucl. Phys. B 83, 217 (1973);

J. Ranft, Fortschr. Phys. 23, 467 (1975)

39. F.W. Büsser et al. (CERN-Columbia-Rockefeller Collaboration), Phys. Lett. B 46, 471 (1973)

40. J.P. Alard et al., Nuovo Cimento A 30, 320 (1975)

41. A.Z. Mekjian, Nucl. Phys. A 312, 491 (1978)

42. J. Gosset, J.I. Kapusta, G.D. Westfall, Phys. Rev. C 18, 844 (1978)

43. A.Z. Mekjian, Phys. Rev. C 17, 1051 (1978)

44. I. Montvay, J. Zimanyi, Nucl. Phys. A 316, 490 (1979)

45. J. Rafelski, M. Danos, Phys. Lett. B 97, 279 (1980)

46. C.B. Chiu, R.L. Heimann, Phys. Rev. D 4, 3184 (1971);

R. Hagedorn, U. Wambach, Nucl. Phys. B 123, 382 (1977) 